INTERNATIONAL JOURNAL OF MULTidisciplinARY RESEARCh AND ANALysis

ISSN(print): 2643-9840, ISSN(online): 2643-9875

Volume 04 Issue 04 April 2021

DOI: 10.47191/ijmra/v4-i4-07, Impact Factor: 6.072

Page No.- $406-412$

\title{
Formation of Neutral Points in the Polarization Characteristics of Secondary Radiation in the Semi-Infinite Medium Model
}

\author{
M.M. Sobirov ${ }^{1}$, J.Yu. Rozikov², V.U. Ruziboyev ${ }^{3}$ \\ $1,2,3$ Fergana State University
}

\begin{abstract}
This article studies the polarization characteristics of diffusely reflected backward radiation when illuminating a semiinfinite, light-scattering medium with unpolarized radiation. The formation of neutral Babine, Brewster and Arago polarization points in the angular polarization characteristics of the secondary radiation is shown. Comparison between the results of exact numerical calculations and approximate analytical formulas has been carried out and physical conditions for the appearance of neutral points depending on the angles of illumination and the multiplicity of scattering have been analyzed.
\end{abstract}

KEYWORDS: Polarized radiation, neutral points, semi-infinite medium, transport equations.

\section{INTRODUCTION}

With the appearance of methods for studying the Earth's surface using polarization probes, interest in studying the transport of polarized radiation in layers of the atmosphere has grown [1,2]. In our papers $[3,4]$, the theory of polarized radiation transfer in media with finite optical thicknesses was outlined. Where, the method of calculation of polarized radiation transfer was developed within the framework of the theory of radiation transfer constructed by Chandrasekar and later generalized by Lenoble and E.L. Ivchenko and others in $[5,6]$. The polarization degrees of diffusely reflected and diffusely passed natural unpolarized solar radiation through atmospheric layers were calculated using this theory. The main attention was removed to the study of the angular characteristics of the Babine, Brewster, and Arago neutral points as a function of the illumination angle, optical thickness, and optical parameters of the medium. The results of these calculations have shown that neutral points are also formed in the semiinfinite medium model. However, in the literature devoted to the study of the characteristics of neutral dots, this issue is not discussed in the semi-infinite medium model and has not been studied consistently.

In contrast to the atmosphere in the light scattering solids or liquids the dielectric permittivity of the medium is high. For this reason research of polarization characteristics of secondary radiation of such media, with the help of this model is of practical value and hopefully will attract the attention of specialists.

In the case of $\tau \rightarrow \infty$ the results of calculations in media with finite optical thicknesses [3], one passes to the model of a semiinfinite medium $[5,6]$. In this case, the intensity and polarization of the reflected backward radiation do not depend on the optical thickness and only the albedo parameter-the quantum yield of the one-time scattering-remains in the solution of the transfer equation. In spite of such simplification, in the characteristics of the reflected backward radiation all features concerning all neutral points observed in the case of a medium with a finite thickness are preserved. The angular characteristics have qualitatively the same character as in the case of a finite-thickness medium for the reflected radiation.

From the theoretical point of view, the study of neutral points in the model of a semi-infinite medium is still interesting because the solution of the integral equations for the $H$-functions by the iteration method has a very high accuracy [5]. This circumstance makes it possible to evaluate the accuracy of the solution of the equations for $X(\mu), Y(\mu)$-functions calculated for a medium with finite thickness. Since in the model of media with finite thickness depending on the value of albedo and optical thickness, the numerical values of the integral equations for $X(\mu), Y(\mu)$-functions have low accuracy, and this was mentioned in [7]. In spite of the fact that various methods have been developed to solve the transfer equation, we have chosen the iteration method to solve the problem at hand. The advantage of this method over others is that the iteration method allows us to estimate the contributions to the intensities and polarizations of the secondary radiation of single-, double-, etc. scattered radiation. This circumstance allows to better understand the process of formation of characteristics of the secondary radiation and to analyze the physical phenomena observed in the medium, such as neutral polarization points. 


\section{Formation of Neutral Points in the Polarization Characteristics of Secondary Radiation in the Semi-Infinite Medium Model}

\section{Problem statement}

Let a plane light wave with total flux $\pi \boldsymbol{F}$ falls on the medium and propagates in the direction $\boldsymbol{\Omega}_{0}\left(\theta_{0}, \varphi_{0}\right)$. Intensity of radiation at any depth $(-z)$ of the medium's plane, propagating in direction $\boldsymbol{\Omega}(\theta, \varphi)$ is determined by solution of transfer equation [6],

$$
\mu \frac{d \mathbf{I}(\tau, \boldsymbol{\Omega})}{\mathrm{d} \tau}=\mathbf{I}(\tau, \boldsymbol{\Omega})-\frac{\widetilde{\omega}_{0}}{4 \pi} \int_{0}^{1} d \mu^{\prime} \int_{0}^{2 \pi} d \varphi^{\prime} \mathbf{P}\left(\boldsymbol{\Omega}, \boldsymbol{\Omega}^{\prime}\right) \mathbf{I}\left(\tau, \boldsymbol{\Omega}^{\prime}\right)-\frac{\widetilde{\omega}_{0}}{4} \exp \left(-\tau / \mu_{0}\right) \mathbf{P}\left(\boldsymbol{\Omega}, \boldsymbol{\Omega}_{\mathbf{0}}\right) \mathbf{F} .
$$

Here the z-axis is perpendicularly upward directed from the incidence plane of the primary flux, $\theta, \varphi$-polar and azimuthal angles, $\tau=\left(\alpha_{a b s}+\sigma\right)$ z-optical thickness of the medium, $\widetilde{\omega}_{0}$ - albedo, is the quantum yield of single scattering, $\boldsymbol{P}\left(\boldsymbol{\Omega}, \boldsymbol{\Omega}^{\prime}\right)$ is a $(4 \times 4)$ matrix of single Rayleigh scattering, the incident flux is also represented as a Stokes column matrix $\mathbf{F}\left(F_{l}, F_{r}, F_{U}, F_{V}\right)$.

Intensities of diffusely reflected back radiation from medium is defined through $\boldsymbol{S}\left(\boldsymbol{\Omega}, \boldsymbol{\Omega}_{\mathbf{0}}\right)$-reflection matrix, which connects with incident flux

$$
\mathbf{I}^{\text {ref }}(\boldsymbol{\Omega})=\left(\widetilde{\omega}_{0} / 4 \mu\right) \mathbf{S}\left(\boldsymbol{\Omega}, \boldsymbol{\Omega}_{\mathbf{0}}\right) \mathbf{F}\left(\tau=0, \overline{\mathbf{\Omega}}_{\mathbf{0}}\right) .
$$

Analytic form of solution for matrix $\boldsymbol{S}\left(\boldsymbol{\Omega}, \boldsymbol{\Omega}_{\mathbf{0}}\right)$ is determined by transfer equation (1), and principles of invariance, and this matrix can be represented as a sum of three independent terms [6],

$$
\mathbf{S}\left(\boldsymbol{\Omega}, \boldsymbol{\Omega}_{\mathbf{0}}\right)=\mathbf{Q}\left\{(3 / 4) \mathbf{S}^{(0)}\left(\mu, \mu_{(1)}\right)+\left[\left(1-\mu^{2}\right)\left(1-\mu_{0}^{2}\right)\right]^{1 / 2} \mathbf{S}^{(1)}\left(\boldsymbol{\Omega}, \boldsymbol{\Omega}_{\mathbf{0}}\right) \mathbf{P}^{(1)}\left(\mu, \varphi,-\mu_{0}, \varphi_{0}\right)++S^{(2)}\left(\boldsymbol{\Omega}, \boldsymbol{\Omega}_{\mathbf{0}}\right) \mathbf{P}^{(2)}\left(\mu, \varphi,-\mu_{0}, \varphi_{0}\right)\right\} .
$$

The analytical form of the solution of these equations is rather cumbersome and for brevity we will not cite them here [3].

\section{Results of numerical calculations}

Usually the value $\mu=\cos \theta$ is used as an angular variable in the radiative transfer equation. Our calculations show that if the geometric angle $\theta$ is used as a variable, the accuracy of the calculation results increases, especially near $\mu \rightarrow 0$. This is due to the fact that in numerical calculations, when performing $\mu$ integration, the space in the angles is not uniformly divided. For example, if we divide the value of $\mu$, in the interval $[0,1]$ at forty points, by the first step of the interval $\Delta \mu=0-0.025$, the angle $\Delta \theta \approx 10^{0}$ corresponds. If we take into account that there is such an uneven distribution of space in $\mu$, it is not difficult to explain why the position of the Arago point is not clearly defined in some works dedicated to the study of the characteristics of neutral points in the atmosphere layers depending on the value of optical parameters of the medium $[8,9,10]$. Since, this point is observed only when the Sun is near the horizon $(\mu \rightarrow 0)$.

The solutions of the problem are defined by eight non-linear integral equations for $X(\mu)$-functions, passing at $\tau \rightarrow \infty$ to $\mathrm{H}$ functions. The solutions of these equations can be determined with high accuracy, by the iteration method, by numerical calculations on a computer.

Let us consider the case where the medium is excited by unpolarized radiation. The stokes parameters of the exciting radiation have the following values

$$
F_{l}=F_{r}=F / 2, \quad F_{U}=F_{V}=0
$$

Due to multiple scattering in the medium, the radiation is polarized and the degree of polarization of the secondary radiation coming out of the medium is determined by the formula

$$
P_{\text {lin }}=\left(I_{l}-I_{r}\right) /\left(I_{l}+I_{r}\right) \text {. }
$$

Fig.1,2 shows the results of the exact calculation of the angular dependence of the polarization of the reflected secondary radiation for two values of albedo: $\widetilde{\omega}_{0}=0.5$ and $\widetilde{\omega}_{0}=0.9$. The polar angles of excitation and observation lie in the same meridional plane $\psi=\varphi_{0}-\varphi=0, \pi$. The source of illumination is in the half-plane $\psi=\varphi_{0}-\varphi=0$. Calculations show that at large values of excitation angles, near the point $\theta_{0} \approx 90^{\circ}$ in the meridional half-plane the Babine point and in the other half-plane the Arago point are observed. As the value of the excitation angle decreases, depending on the albedo value, from some angle $\tilde{\theta}_{0} \approx \theta_{0}$, the Arago point disappears, but a Brewster point appears near the Babine point. When $\widetilde{\omega}_{0}=0.5$, the value of this angle is $\tilde{\theta}_{0} \approx 82^{0}$. As the albedo value increases, the Arago point is observed at larger angles of illumination. At $\widetilde{\omega}_{0}=0.9$ the value of this angle is $\tilde{\theta}_{0} \approx 72^{0}$. With decrease of the excitation angle value $\theta_{0}$, the Babine and Brewster points shift towards the zenith point, and the angular distance between them first increases to $\theta_{0} \approx 50^{\circ}$, and then decreases and merges into one point, at $\theta_{0}=0^{0}$. Increasing the albedo value, also leads to widening of the angular distance between these points. At the same time the polarization amplitude increases in the area between the neutral points, but in the negative part of the characteristics, the polarization decreases. The polarization maxima correspond to the point where the propagation directions of the incident and reflected radiation are mutually perpendicular. The polarization maximum between the neutral points of Babine and Brewster corresponds to the angle of incidence of the incident radiation, i.e. the maximum is observed when the incident radiation is reflected backward. This behavior 


\section{Formation of Neutral Points in the Polarization Characteristics of Secondary Radiation in the Semi-Infinite Medium Model}

of the neutral points is similar to the pattern observed for reflected radiation in the model of media with finite thickness $[4,9,10]$. Figures 1 and 2 also show the results of the calculation of the angular characteristic of the degree of polarization of the secondary radiation, from the multiplicity of scattering for two values of albedo. The calculations in Figure 1 were performed for the scattering medium at $\widetilde{\omega}_{0}=0.5$, when the values of the absorption and scattering coefficient of the medium are equal to $\sigma=$ $\alpha_{a b s}$. The graphs show that for the formation of neutral points in the polarization characteristics of the secondary radiation, a twofold scattering of the primary radiation in the medium is sufficient. Increasing the number of scatterings leads to increasing the value of polarization of radiation between neutral points, and to decreasing the maximum polarization.

In Fig. 2 the calculations are performed in the case of a highly scattering medium, when $\sigma=9 \alpha_{a b s}$ and $\widetilde{\omega}_{0}=0.9$, the values of the scattering coefficient are much larger than the absorption coefficient value. In this case, the degree of polarization in the region between the neutral points is noticeably higher than in Fig. 1, and depolarization increases in the region of negative polarizations.

The results of these calculations show that when the medium is excited by unpolarized radiation, across the meridional plane of incidence and observation, the difference between the $I_{l}, I_{r}$ components of the intensity of the reflected radiation, changes sign twice.

For verification, the results of the analytical calculation of the angular characteristics of the degree of polarization in equations (1)-(3), in the case of single scattering, are given below,

$$
P_{\text {lin }}=\frac{2 \alpha^{2}-\left(1-\mu_{0}^{2}\right)\left(1-\mu^{2}\right)+4 \mu \mu_{0} \alpha \cos \left(\varphi_{0}-\varphi\right)-\left(1-\mu_{0}^{2}\right)\left(1+\mu^{2}\right) \cos 2\left(\varphi_{0}-\varphi\right)}{2 \alpha^{2}-\left(1-\mu_{0}^{2}\right)\left(1-\mu^{2}\right)+4 \mu \mu_{0} \alpha \cos \left(\varphi_{0}-\varphi\right)-\alpha^{2} \cos 2\left(\varphi_{0}-\varphi\right)},
$$

where, $\alpha=\left(\left(1-\mu_{0}^{2}\right)\left(1-\mu^{2}\right)\right)^{1 / 2}, \varphi_{0}, \varphi$ are the azimuthal angles of the incident and scattered radiation. The results of the calculation of the angular characteristics of polarization in the first iteration, calculated by the exact (3) and by the analytical forum (5), coincide with high accuracy.

Figure 3 shows the angular characteristics of the secondary radiation intensity, for two values of albedo. The graphs show that in the geometry of normal incidence and reflection back $\theta_{0}=\theta=0$, the intensity of secondary radiation is maximal. As the excitation angle deviates from the normal, the intensity maximum shifts toward the horizon. As the value of quantum yield of single scattering increases, the intensity of secondary radiation increases at all angles of illumination and observation. At the polarization rotation points, the intensity also changes values modulo.

Below we discuss the case when the medium is excited by linearly polarized radiation, the polarization plane of the incident and reflected radiation lie in the same meridional plane, containing angle $45^{0}$ between the $F_{l}, F_{r}$ components, the phase difference of oscillations between them is equal to zero. The stokes parameters of the excitation radiation have the following values

$$
F_{l}=F_{r}=F / 2, F_{U}=F, F_{V}=0 .
$$

The degree of polarization of radiation is determined by the formula

$$
P_{\text {lin }}=U /\left(I_{l}+I_{r}\right) \text {. }
$$

Angular polarization characteristics of secondary radiation for different values of the excitation angle, with albedo $\widetilde{\omega}_{0}=0.5$ and $\widetilde{\omega}_{0}=0.9$ are shown in Fig. 4,5. It can be seen from figures that in contrast to case of excitation of medium by non-polarized radiation, in this case only one neutral point is observed in angular polarization characteristics of reflected radiation. As one would expect, this point corresponds to the point where the directions of the incident and reflected radiation are mutually perpendicular. At the first iteration by the general formula (3), we can introduce the formula for calculation of polarization for the once scattered radiation

$$
P_{l i n}=\frac{2\left(2 \alpha \cos \left(\varphi_{0}-\varphi\right)-2 \mu_{0} \mu \cos 2\left(\varphi_{0}-\varphi\right)\right)}{2 \alpha^{2}-\left(1-\mu_{0}^{2}\right)\left(1-\mu^{2}\right)+4 \mu \mu_{0} \alpha \cos \left(\varphi_{0}-\varphi\right)-\alpha^{2} \cos 2\left(\varphi_{0}-\varphi\right)}
$$

Figure 6 shows the angular characteristics of the secondary radiation intensity, for two values of albedo. As the albedo value increases, the intensity of the radiation exiting the medium decreases, since the multiplicity of scatterings increases. At the zero points of polarization the phase difference between the components $I_{l}, I_{r}$ changes sign, this leads to a change in the sign of the intensity.

\section{CONCLUSIONS}

1. Theoretically investigated the angular polarization characteristics of diffusely reflected secondary radiation when illuminating a light-scattering, semi-infinite medium with unpolarized and polarized radiation. 


\section{Formation of Neutral Points in the Polarization Characteristics of Secondary Radiation in the Semi-Infinite Medium Model}

2. It is shown that in the model of a semi-infinite medium in the angular polarization characteristics of diffusely reflected radiation neutral points, similar to those discussed in atmospheric physics, are formed in the case of media with finite thicknesses. Positions of these points strongly depend on value of quantum yield of single scattering-albedo and angle of illumination.

3. The conditions for the formation of neutral points depending on the multiplicity of scattering were investigated. It is shown that for the formation of neutral points, a double scattering of the incident radiation in the medium is sufficient.

4. Calculations show that this polarization behavior has a general character, and, unlike turbid media, neutral points can also be observed in light-scattering media with high dielectric permittivity, both in liquids and solids.

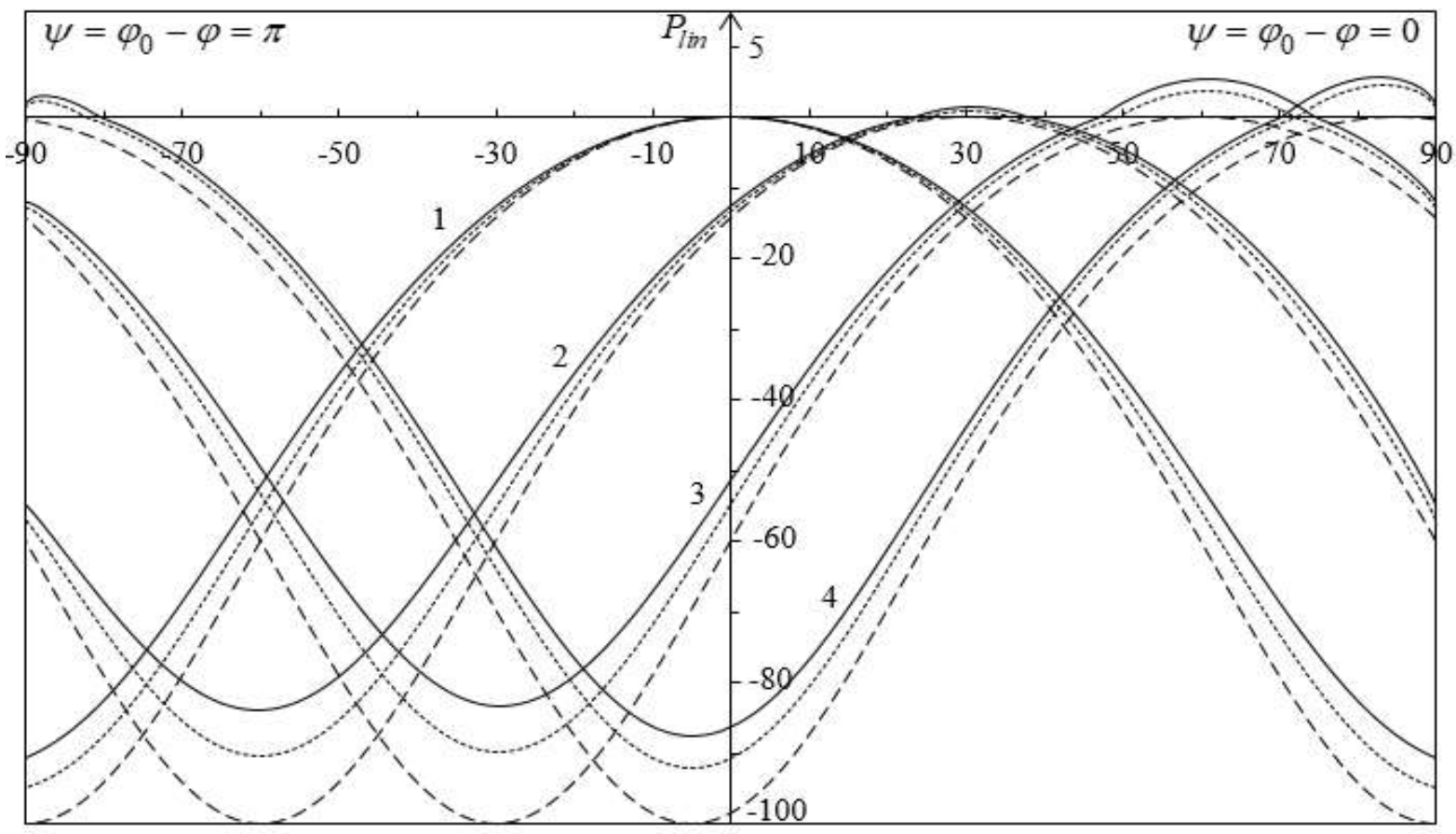

Fig.1. Dependences of polarization of reflected backward radiation on the multiplicity of scattering, in case $\widetilde{\omega}_{0}=0.5$. Dotted curves describe single scattering, dotted double scattering, bold curves the results of exact scattering. Illumination angles: $\theta_{0}=$ $0^{0}$ (cr. 1), $\theta_{0}=30^{\circ}(2), \theta_{0}=60^{\circ}(3), \theta_{0}=85^{0}(4)$.

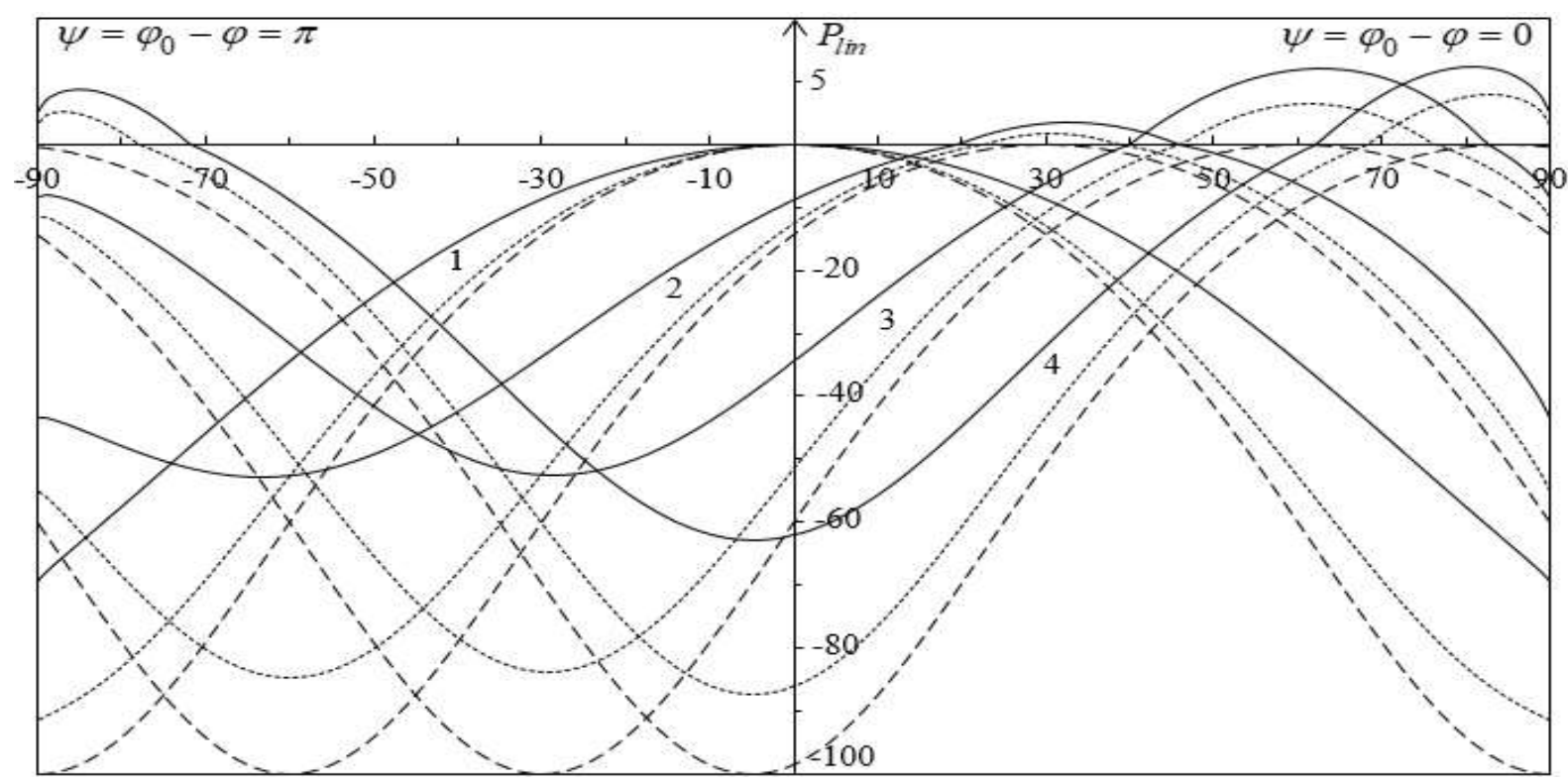

Fig.2. Dependences of polarization of the secondary radiation on the multiplicity of scattering, in case $\tilde{\omega}_{0}=0.9$. Dotted curves describe single scattering, dotted double scattering, bold curves the results of exact scattering. Illumination angles: $\theta_{0}=0^{0}$ (cr. 1), $\theta_{0}=30^{\circ}(2), \theta_{0}=60^{\circ}(3), \theta_{0}=85^{\circ}(4)$. 
Formation of Neutral Points in the Polarization Characteristics of Secondary Radiation in the Semi-Infinite Medium Model

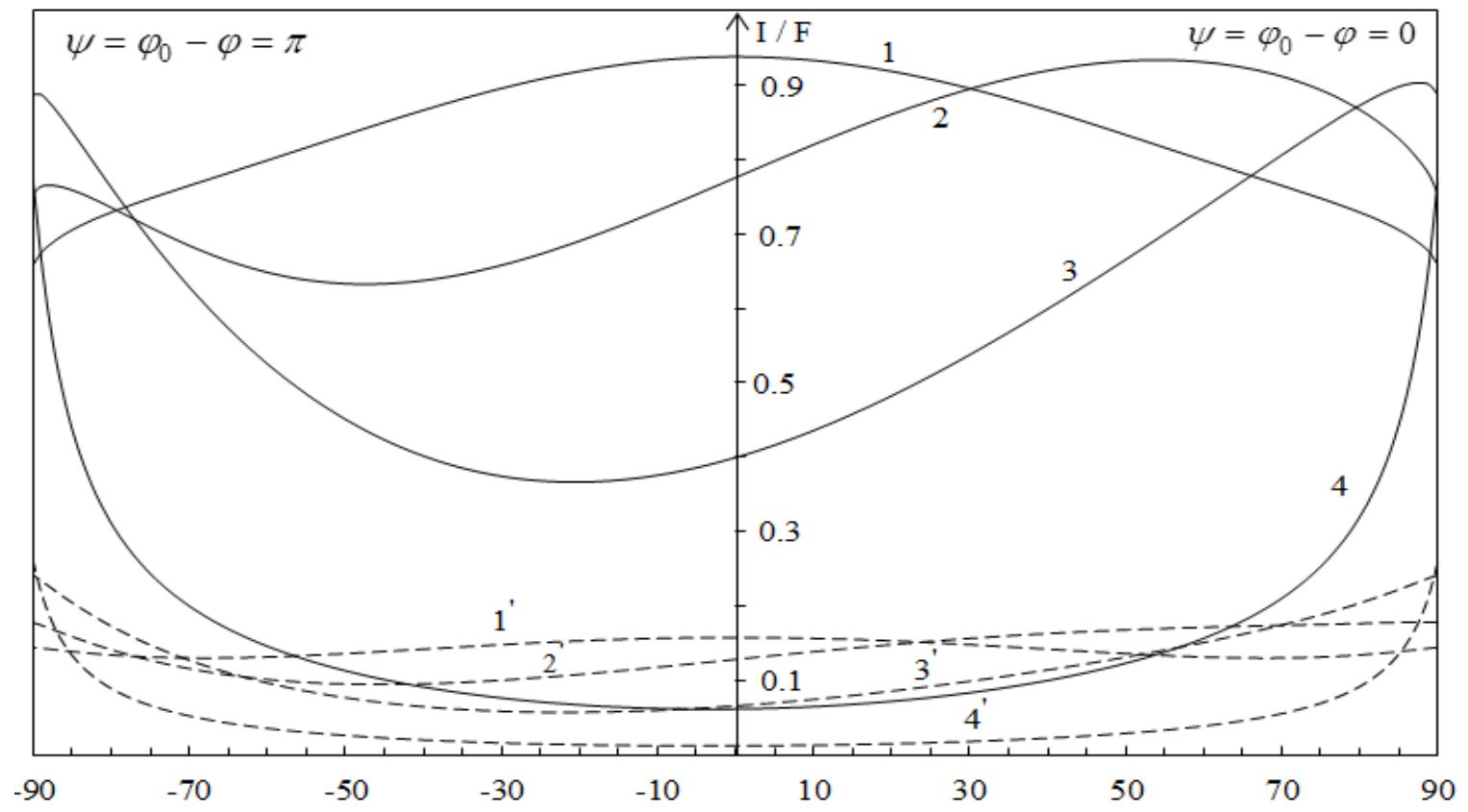

Fig.3. Angular dependences of the intensity of secondary radiation for two values of albedo. The dotted curves are calculated at $\widetilde{\omega}_{0}=1 / 3$, the bold ones at $\widetilde{\omega}_{0}=0.9$. Illumination angles: $\theta_{0}=0^{\circ}\left(\mathrm{cr} \cdot 1,1^{\prime}\right), \theta_{0}=30^{\circ}\left(2,2^{\prime}\right), \theta_{0}=60^{\circ}\left(3,3^{\prime}\right), \theta_{0}=85^{0}(4)$.

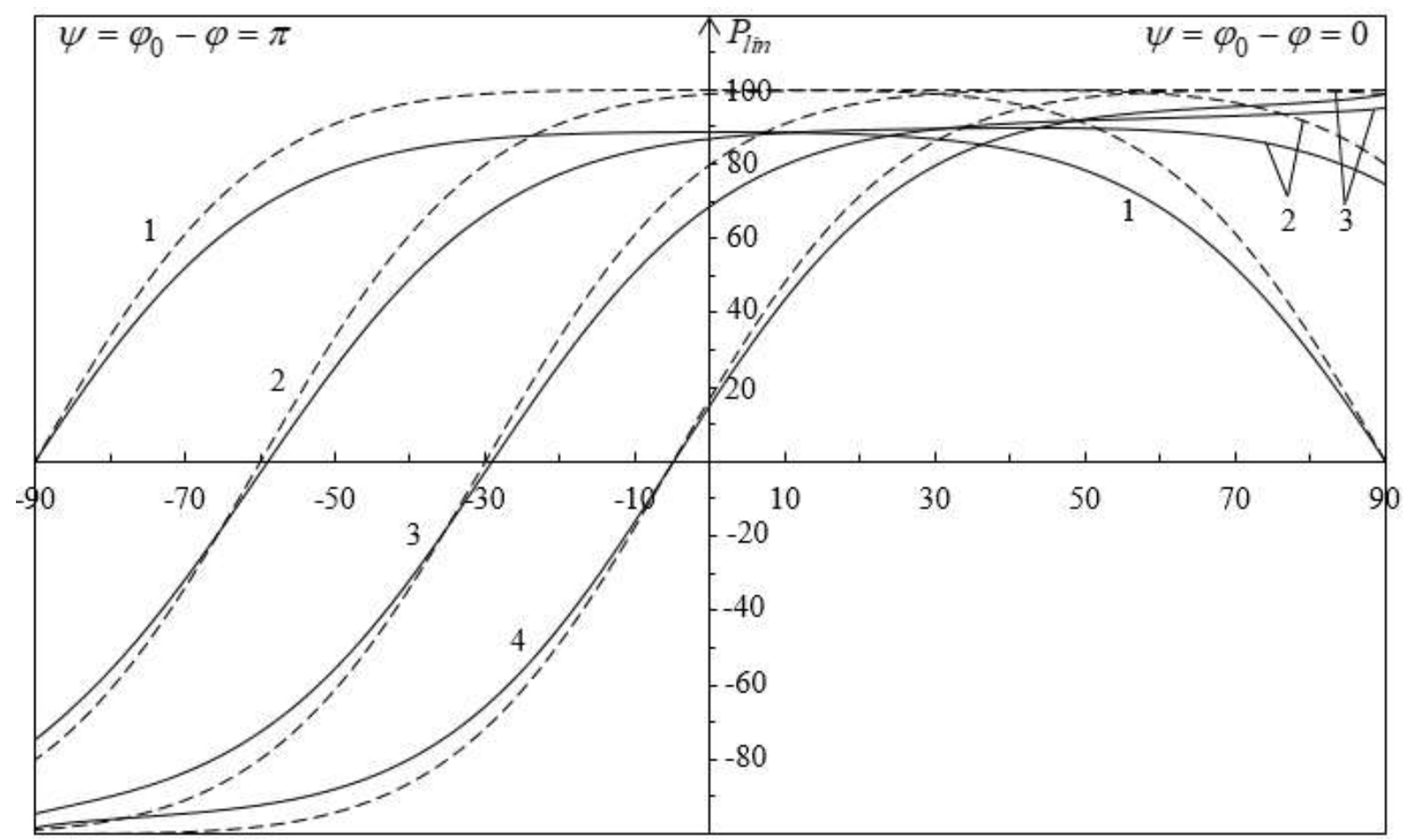

Fig.4. Dependences of polarization of reflected radiation on multiplicity of scattering, at excitation by linearly polarized light. Dotted curves single scattering, bold results of exact scattering, $\tilde{\omega}_{0}=0.5$. Illumination angles: $\theta_{0}=0^{0}$ (cr. 1$), \theta_{0}=30^{\circ}(2)$,

$$
\theta_{0}=60^{\circ}(3), \theta_{0}=85^{\circ}(4) \text {. }
$$


Formation of Neutral Points in the Polarization Characteristics of Secondary Radiation in the Semi-Infinite Medium Model

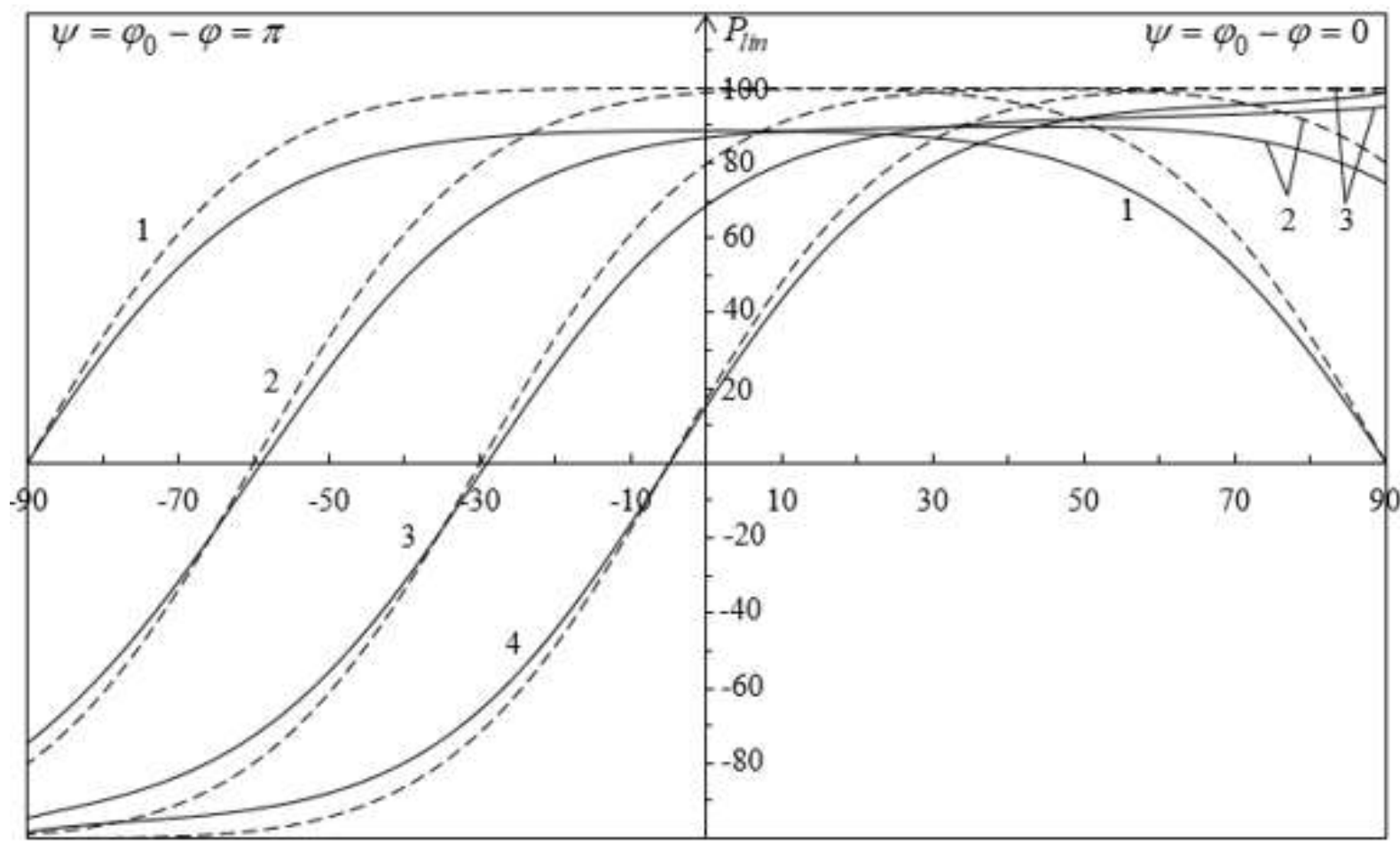

Fig.5. Dependences of polarization of the secondary radiation on the scattering multiplicity, in the case $\widetilde{\omega}_{0}=0.9$. The dotted curves describe the single scattering, the bold curves the results of the exact calculation. Illumination angles: $\theta_{0}=0^{0}$ (cr. 1), $\theta_{0}=30^{\circ}(2), \theta_{0}=60^{\circ}(3), \theta_{0}=85^{\circ}(4)$.

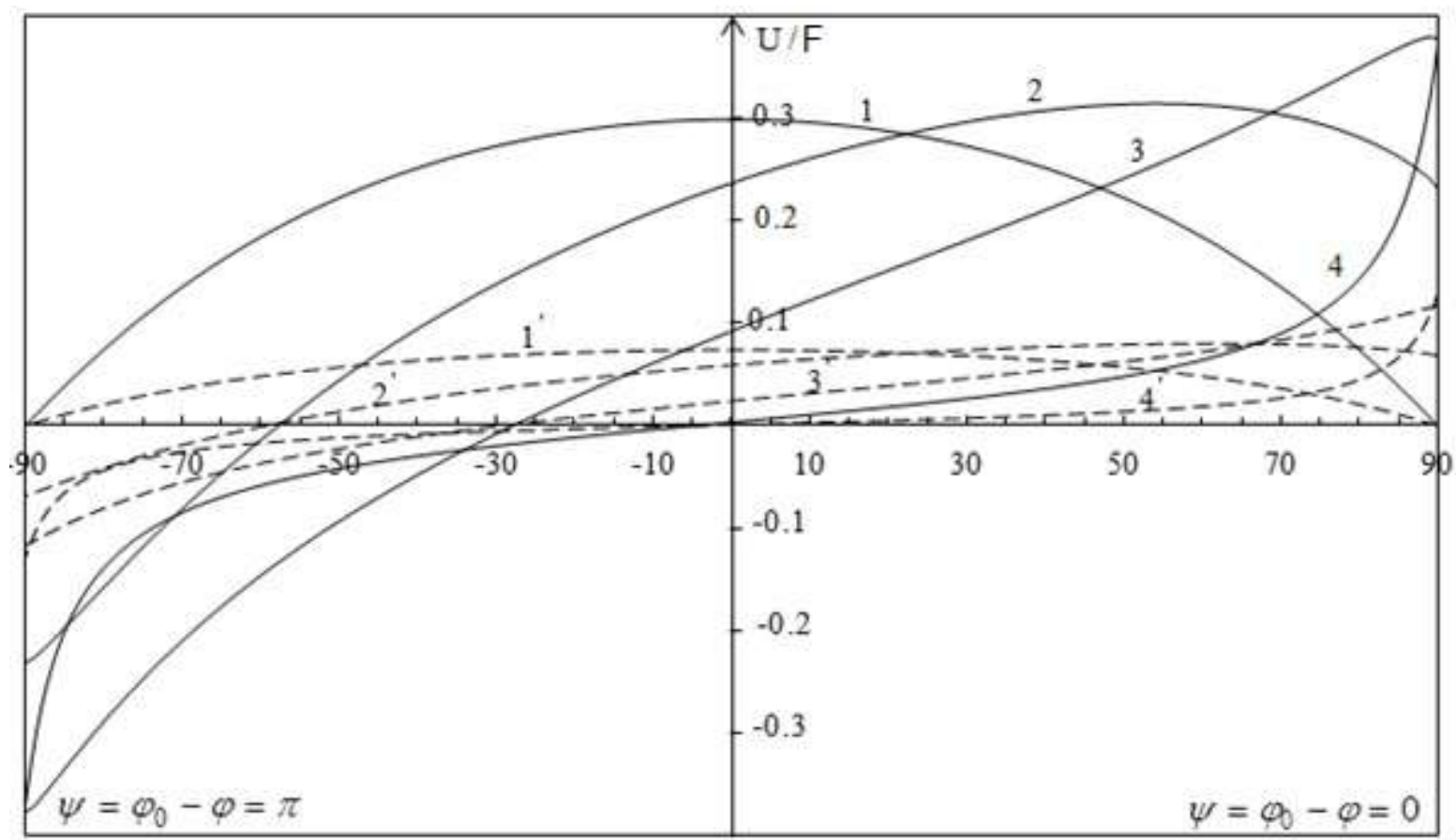

Fig.6. Angular dependences of the intensity of secondary radiation for two values of albedo. The dotted curves are calculated at $\widetilde{\omega}_{0}=1 / 3$, the bold ones at $\widetilde{\omega}_{0}=0.9$. Illumination angles: $\theta_{0}=0^{0}\left(\mathrm{cr} .1,1^{\prime}\right), \theta_{0}=30^{0}\left(2,2^{\prime}\right), \theta_{0}=60^{0}\left(3,3^{\prime}\right), \theta_{0}=85^{\circ}(4)$

\section{REFERENCES}

1) Сушкевич Т.А. // Материалы пятнадцатой открытой всероссийской конференции. “Современные проблемы дистанционного зондирования Земли из космоса". 2017 г., Москва, Россия. С.88 - 117 Институт космических исследований РАН, 2017 (2008).

2) Lei Y., Taxia W., Xuegi W. // Understanding of Atmospheric Systems with Efficient Numerical Methods for Observation 
Formation of Neutral Points in the Polarization Characteristics of Secondary Radiation in the Semi-Infinite Medium Model

and Prediction. 2017. Chapter 3, P.23-49. DOI: 10.5772/intechopen.79937.

3) Собиров М.М., Розиков Ж.Ю. // Научно-технический журнал, Ферганский ПИ. 2020. 24, 4, стр.15-24.

4) Собиров М.М., Розиков Ж.Ю. // Узбекский физический журнал, АН РУзб. Ташкент, (принято в печать) 2021.

5) Lenoble J.// Journal of Quantativ Spektroskopy and Radiativ Transfer. 1970. V.10. P. 372-378.

6) Ивченко Е.Л., Пикус Г.Е., Юлдашев Н.Х. // Журнал экспериментальной и теоретической физики. 1980. Т. 79,С. 15731590.

7) Vijay N., King-Fai L., Yuk L.Y. // The Astrophysical Jornal. 2009. P. 1909-1920 doi:10.1088/0004-637X/691/2/1909.

8) Чандрасекар С. Перенос лучистой энергии. М. : Иностранная литература, 1953. 432 с.

9) Coulson, K. L. Polarization and intensity of light in the atmosphere. Deepak Publishing : 1988. A.

10) Kattawar G.W., Plass G.N., Hitzfelber S.J. //Appleid Optics. 1976. V.15(3), P.632-647. DOI:10.1364/AO.15.000632) 